\title{
Refractive Index Lithography Materials Based on Chemically Amplified Reaction
}

\author{
Takashi Yamashita, Atsushi Nara, Tomomi Fujimoto and Kunihiko Okano \\ Department Pure and Applied Chemistry, Faculty of Science and Technology, \\ Tokyo University of Science, 2641 Yamazaki, Noda, Chiba 278-8510, Japan \\ yama@rs.noda.tus.ac.jp
}

This article is dedicated to late Dr. Ito for his distinguished invention, fantastic technology, and warmhearted humanity.

Refractive index lithography was performed on polymer films consist of a photo-acid generator (PAG), t-BOC-protected naphthazarine as an acid chromic dye. The key reaction is the chemically amplified de-protection of the t-BOC group, by which color of the material change followed by refractive index change. Naphthazarine-containing polymer was also prepared. The induced refractive index was as large as $4 \times 10^{-3}$, which is enough large to apply to optical waveguide fabrication.

Keywords: refractive index lithography / photo optical effect / naphthazarine/ chemical amplification

\section{Introduction}

Developing those materials whose refractive index can be directly patterned with lithographic technique is important for fabricating photonic devices, optics, optical waveguides, 3D-optical data storage media, optical computing devices and so on." Recently we have been developing various photo-optical materials, whose refractive indices are controlled by photochemical reactions. The photo-optical effect involves photochromic reaction of azobenzenes, ${ }^{2)}$ norobornadienes, ${ }^{3)}$ photo-rearrangement of sulfonates, ${ }^{4)}$ and two-photon-sensitized reaction ${ }^{5)}$ and so on. Change in the chemical structure during photochemical reaction is usually followed by the absorption change due to the electronic state change. And then refractive index also changes following to Kramas-Kronis's relationship. Thus, photo-optical effect is the technique that is combination of photochemistry and optical properties of the materials.

Since efficiency of the photoreaction of those materials is limited to the quantum yield of the photochemical reaction of the materials, however, involving chemical amplification reaction is desirable for improving the photo-optical effect efficiency . Thus we designed a novel system which combines a PAG and t-BOC protected naphthazarine. The naphthazarine is a dye whose color is based on

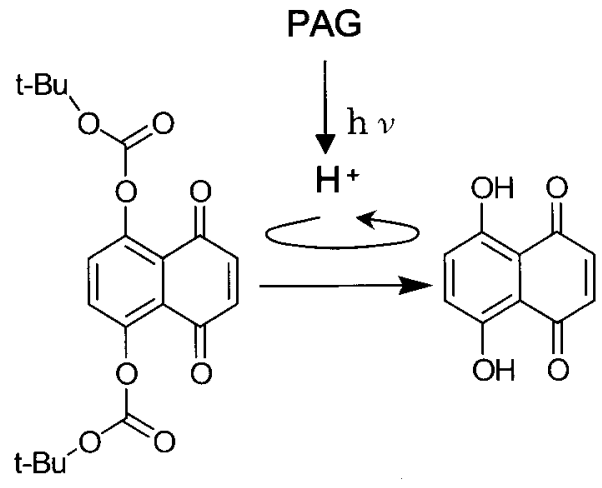

Scheme 1 Chemically amplified photo-optical effect of $\mathrm{t}$-BOC protected naphthazarine 


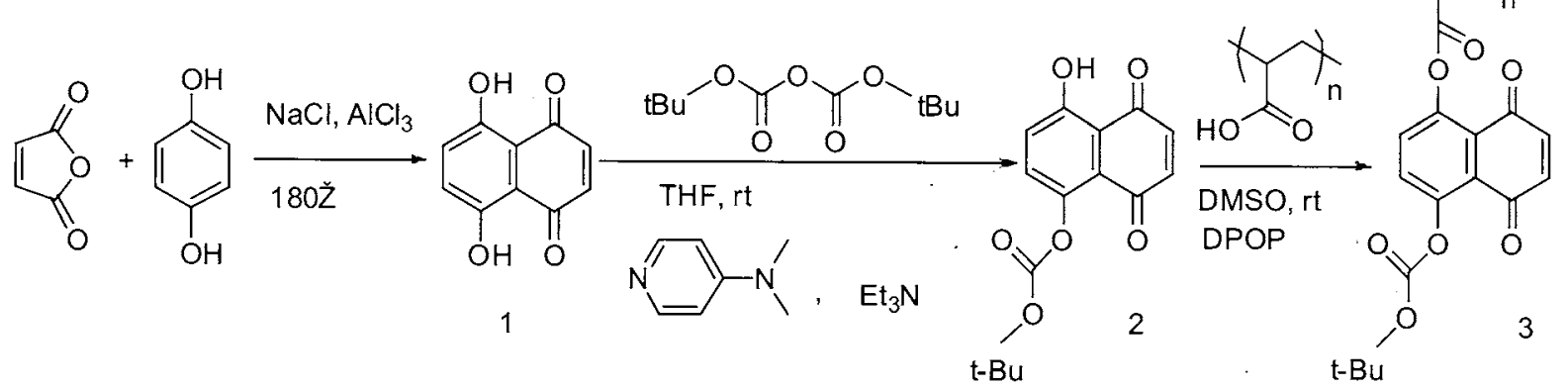

Scheme 2 Preparation of t-BOC protected Naphthazarine, and polymer-bound t-BOC protected Naphthazarine

intramolecular conjugation involving hydrogen bonding between the hydroxyl group and quinine group. Therefore the electronic state of the naphthazarine should be controlled by the de-protection of $\mathrm{t}-\mathrm{BOC}$ group with chemically amplified reaction in Scheme 1.

\section{Experimental}

\subsection{Materials}

Naphthazarine was prepared by the reaction of hydroquinone and maleic anhydride, which was then treated by di(tert-buthyl) carbonate to give t-BOC protected naphthazarine. Naphthazarine-containing polymer films were prepared by spin-coating of the mix solution of the $\mathrm{t}$-BOC protected naphthazarine (2wt \%), a photo acid generator, NAI-105 (1 wt \%), and PMMA. Polymer-bound naphthazarine was prepared by the reaction of the naphthazarine and poly (acrylic acid) in the presence of a dehydration reagent, followed by repeated reprecipitation.

Photo-dynamics and photophysical process measurement was performed by electron beam pulse radiolysis with a linear accelerator (acceleration energy of $35 \mathrm{MeV}$ ), and a pulsed Xe lamp (pulse duration of $1 \mu \mathrm{sec})$. Laser flash photolysis was performed with a Nd:YAG laser (10 nsec, third harmonics) as pump source and $\mathrm{cw}$-Xe lamp as a monitor lamp.

Photochemical reaction was performed by photo irradiating $350 \mathrm{~nm}$ light from ultra-high pressure mercury lamp (Ushio SX-UI501HQ) using a monochromator.

UV/Vis absorption spectra were recorded on a Jasco V-550 spectra photometer.

Refractive indices of the samples were determined by a prism coupled m-line measurement system using $633 \mathrm{~nm}$ light form a He-Ne laser as the probe.

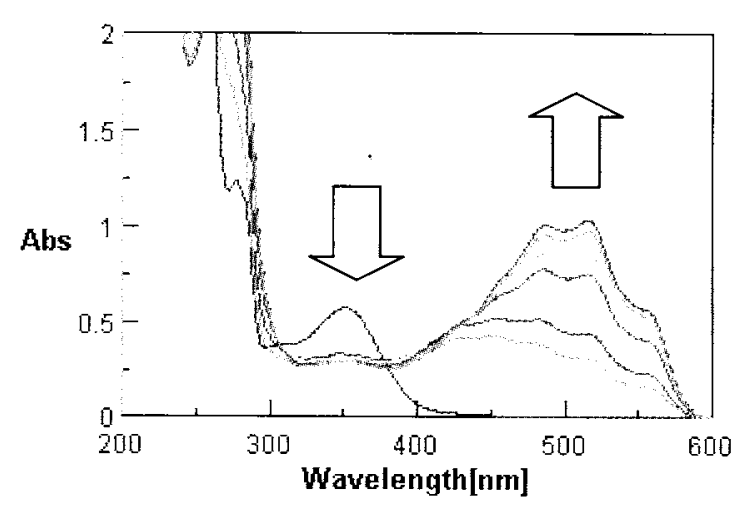

Figure 1 Change in UV spectra of t-boc naphthazarine in THF solution heated at $100^{\circ} \mathrm{C}$

\section{Results and Discussion}

3.1 Photoirradiation of Naphthazarine solution.

Fig 1 shows the spectral change of t-BOC protected naphthazarine solution in the presence of a PAG under thermal treatment after photoirradiation. Absorption with maximum at $430 \mathrm{~nm}$ appeared until $5 \mathrm{~min}$ thermal treatment, and then the absorption maximum shifted to $515 \mathrm{~nm}$ after then. This observation shows the de-protection of the $\mathrm{t}-\mathrm{BOC}$ protect naphthazarine occurs in two steps, in which one t-BOC group eliminates within 5 min under that condition and then the other $\mathrm{t}-\mathrm{BOC}$ reacts.

Similar absorption change was observed for the naphthazarine doped in PMMA film under thermal treatment at $100^{\circ} \mathrm{C}$ after photoirradiation. Fig. 2 shows $\mathrm{m}$-line spectra of the photoirradiated sample. Seven guided modes were observed, with which refractive index of the sample film can be determined. The refractive indices of the film before and after photoirradiation are listed in Table 1 


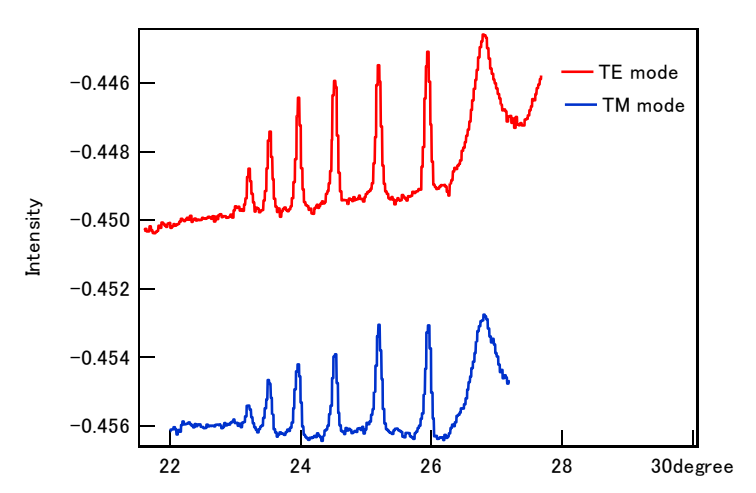

Figure 2 m-line spectra of PMMA film containing $2 \mathrm{wt} \%$ t-boc naphthazarine and $1 \mathrm{wt} \%$ PAG

Table 1. Refractive index of sample film before and after photo-irradiation

\begin{tabular}{llll}
\hline mode & $\begin{array}{l}\text { Before } \\
\text { Irradiation }\end{array}$ & $\begin{array}{l}\text { After } \\
\text { Irradiation }\end{array}$ & $\Delta \mathrm{n}$ \\
\hline TE & 1.49076 & 1.49069 & $-1 \times 10^{-4}$
\end{tabular}

$\mathrm{TM}$ $1.49060 \quad 1.49102$

$$
4 \times 10^{-4}
$$

Induced refractive index is as large as $4 \times 10^{-3}$ for TM mode, which is large enough to optical waveguide manufacture. Refractive index of materials is due to polarization, and refractive index and absorption correspond to real and imaginary part of the polarization. Those absorption and refraction are related by Kramas-Kronig's relation each other, where $\mathrm{n}$ and $\mathrm{\kappa}$ are the refraction and distinction coefficients, $\omega$ is the observation wavelength, and $P$ is principal value, respectively.

$$
\begin{aligned}
& n(\omega)-1=\frac{2}{\pi} P \int_{0}^{\infty} \frac{\omega_{1} \kappa\left(\omega_{1}\right)}{\omega_{1}^{2}-\omega^{2}} d \omega_{1} \\
& \kappa(\omega)=-\frac{2}{\pi} P \int_{0}^{\infty} \frac{\omega n\left(\omega_{1}\right)}{\omega_{1}^{2}-\omega^{2}} d \omega_{1}
\end{aligned}
$$

Spectral shift of the sample film after photo-irradiation and thermal treatment should be the reason for the increase in the refractive index at $633 \mathrm{~nm}$.

3.2 Photo-optical effect of polymer-bound naphthazarine film.

Figure 3 shows the spectral change of the polymer-bound naphthazarine bulk film after photoirradiation of $401 \mathrm{~nm}\left(220 \mu \mathrm{W} / \mathrm{cm}^{2}\right)$ light and following thermal treatment at $100^{\circ} \mathrm{C}$. The polymer-bound naphthazarine is chemically amplified de-protected to show a new absorption at $550 \mathrm{~nm}$. The new absorption due to naphthazarine is strucureless and without peak shift because the naphthazarine is mono-substituted.

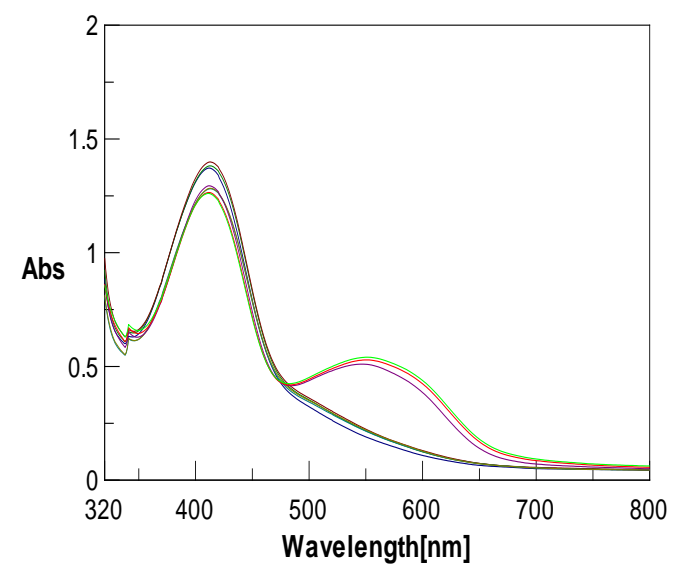

Figure 3 Spectral change of polymer-bound t-BOC protected naphthazarine film containing $1 \mathrm{wt} \%$ PAG under thermal treatment at $100^{\circ} \mathrm{C}$ after photoirradiation.

Refractive indices of the film are 1.6302 for TE mode, and 1.6300 for TM mode, respectively. The average refractive index increases from 1.6301 to 1.6463 after photoirradiation, whose induced refractive index is as large as $16 \%$.

3.3 Photophysical process of the photo-optical materials.

In order to clarify the photophysical process of the photo optical material with extraordinary large refractive index induction, photochemistry of the PAG, NAI-105, was measured. Quantum yield of photo decomposition of the PAG was 0.022 in THF solution shown in Figure 4. On the other hand the quantum yield of the NAI-105 in solid state PMMA has wide distribution ranging from 0 to 0.14 due to

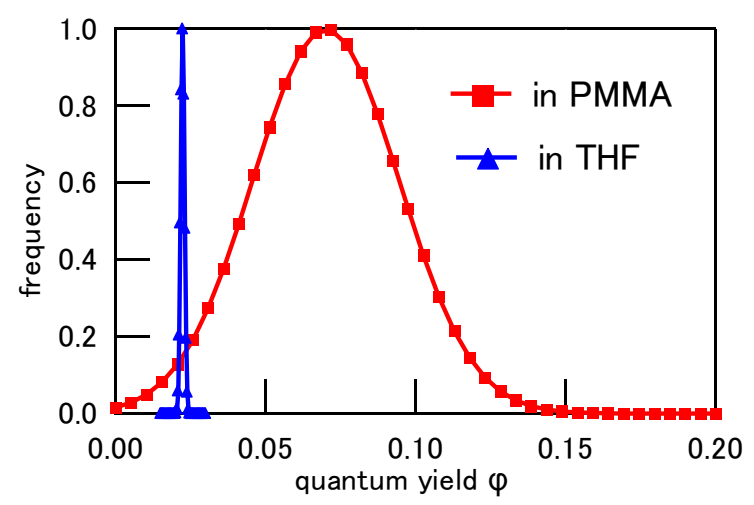

Figure 4 Quantum yield distribution of PAG in THF solution and in solid state PMMA 
inhomgeniety of free volume distribution in PMMA.

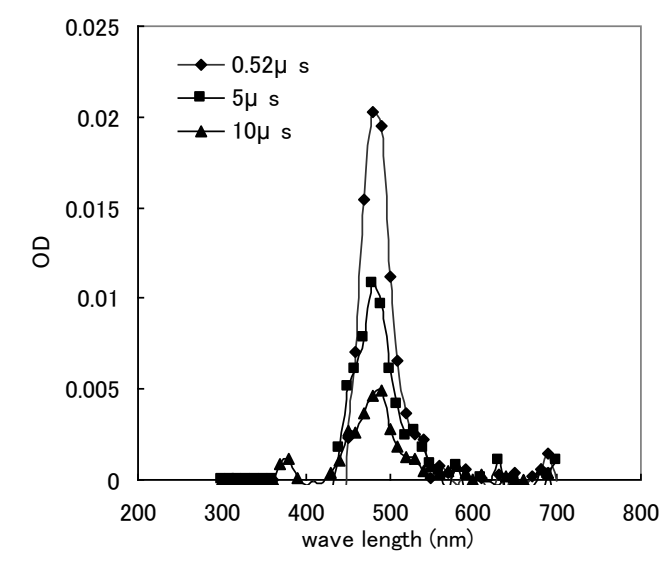

Figure 5 Transient absorption spectra of NAI-105 in $\mathrm{CH}_{2} \mathrm{Cl}_{2}$ by laser flash photolysis

Figure 5 shows transient absorption spectra of the NAI-105 measured by laser flash photolysis in $\mathrm{CH}_{2} \mathrm{Cl}_{2}$ solution. The absorption maximum is $480 \mathrm{~nm}$ and the lifetime is $7 \mu \mathrm{sec}$.

In order to determine the active species in the laser flash photolysis, electron beam pulse radiolysis of the sample was performed in benzene, THF, and $\mathrm{CH}_{2} \mathrm{Cl}_{2}$ solution (Figure 6). Absorption of radical anion of NAI-105 is observed at $680 \mathrm{~nm}$ in THF solution, and that of radical cation is at $560 \mathrm{~nm}$ in $\mathrm{CH}_{2} \mathrm{Cl}_{2}$ solution. Electronic excited state (T-T absorption) is also observed at $480 \mathrm{~nm}$ in the electron beam pulse radiolysis experiment in benzene solution. Therefore the active species of the observed transient absorption can be assigned as T-T absorption of NAI-105. Thus the rate determining step of the acid generation

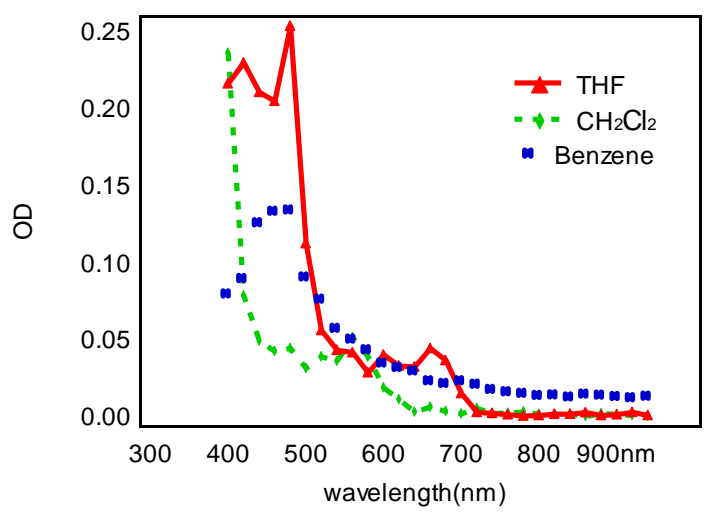

Figure 6 Transient absorption spectra of NAI-105 in THF or $\mathrm{CH}_{2} \mathrm{Cl}_{2}$ solution by electron beams pulse radiolysis

reaction of NAI-105 is as long as $7 \mu$ sec compared to the salvation dynamics of several nsec. This is quite different from the photochemical reaction of some photochromic compounds such as azobenzene and so on, whose lifetime is as short as several ps. Those compounds with shorter lifetime in their rate determining steps preserving static free volume around them even in solution, while the free volume around NAI-105 should be dynamic one in solution. Therefore NAI-105 preserves no more sufficient free volume for the photoreaction in solution that the reaction in solid state PMMA becomes preferable.

\section{Conclusions}

Chemically amplified photo-optical effect of t-BOC protected naphthazarine was performed to induce $16 \%$ of refractive index change with polymer-bound sample. The lifetime of the PAG in the rate-determining step was found to be $7 \mu \mathrm{sec}$, and the quantum yield for photoreaction in much preferable in PMMA matrix, due to preservation of static free volume. Those materials are used for refractive index lithography for waveguide or optics fabrication. And the chemical amplification is the key step for the increase in the reaction efficiency of the photo-optical effect.

\section{References}

1) S.Kawata, et.al.,O plus E.,20,1044(1998)

2) S. Morino, S. Machida, T. Yamashita, K. Horie, $J$. Phys. Chem., 99 (25), 10280-10284 (1995)

3) Y Sato, J Kato, T Yamashita, J. Photopolymer Sci. Tech., 17, 291-296 (2004)

4) S. Morino, T. Watanabe, Y. Magaya, T. Yamashita, K. Horie and T. Nishikubo, J. Photopolym. Sci. Technol., 7, 121-126 (1994)

5) J. Kato, K. Yuasa, Y. Maekawa, M. Yoshida, T. Yamashita, RadTech Asia 03, proceedings 328-331 (2003)

6) K. Yuasa, K. Enomoto, Y. Maekawa, J. Kato, T. Yamashita, M. Yoshida, J. Photopolymer Sci. Tech., 17, 21-28 (2004)

7) J. Kato, K. Yuasa, H. Matsushita, Y. Maekawa, K. Enomoto, T. Ishii, K. Ito, T. Yamashita, $J$. Photopolym. Sci. Tech., 19, 105-110 (2006)

8) T. Yamashita, S. Miake, M. Ogawa, J. Kato, Y. Maekawa, K. Enomoto, Y. Muroya, K. Katsumura, $J$. Photopolym. Sci. Tech., 19, 117-122 (2006)

9) H. Onozeki, J Kato, Y Maekawa, Y Muroya, Y 
Katsumura, T. Yamashita RadTech Asia 03 proceedings, 618-621 (2003)

10) S. Shinoda, J. Kato, T. Yamashita, J. Photo PolymerSci. Technol., 16, 101-106 (2003)

11) J. Kato, Y. Muroya, Y. Katsumura, T. Yamashita, Radtech Asia 03(2003.12) proceedings pp 332-335(2003)

12) M. J. Plater, T. Jackson, Tetrahedron, 59, 4673-4685 (2003)

13) H. Irngartinger, R. Herpich, Eur. J. Org. Chem., 595-604 (1998)

14) I. Brehm, S. Hinneschiedt, H. Meier, Eur. J. Org. Chem., 3162-3170 (2002)

15) R. W. Lenz, A. Furukawa, P. K. Bhowmik, R. O. Garay, and J. Majnusz, Polymer, 32, 1703-1712 (1991)
16)M. Weck, B. Mohr, B. R. Maughon, R. H Grubbs ,Macromolecules, 30, 6430-6437 (1997)

17)J. Manca, L. Lutsen, T. J. Cleij, D, Vanderzande, Macromol. Chem. Phys., 208, 196-206 (2007)

18)A. J. J. M. van Breemen, P. T. Herwig, C. H. T. Chlon, J. Sweelssen, H. F. M. Schoo, E. M. Benito, D. M. de Leeuw, C. Tanase, J. Wildeman, P. W. M. Blom, Adv. Funct. Mater., 15, $872-876$ (2005)

19) DJ.B.Bruce, A.JS.Sorrie et al. Journal of Chemical Society. 1953, 2403-6.

20) J -M. Kim, J.-H. Kang, D.-K. Han, C.-W. Lee, K.-D. Ahn, Chem. Mater. 1998, 10, 2332.

21) F. Ortica, J. C. Scaiano, G. Pohlers, J .F. Cameron, and A. Zampini, Chem. Mater. 2000, 12, 414-420 